\title{
Perspectivas de actores clave respecto del acuerdo binacional entre Argentina y Chile sobre la erradicación del castor norteamericano y la restauración de los ecosistemas afectados
}

\author{
Perspectives of key stakeholders on the bi-national agreement between Argentina and Chile \\ concerning the eradication of North American beavers and the restoration of affected ecosystems
}

\author{
Christopher B Anderson ${ }^{\text {a,b,*, }}$, Catherine Roulier a,c, J Cristóbal Pizarro a,d

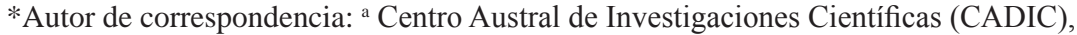 \\ Consejo Nacional de Investigaciones Científicas y Técnicas (CONICET), Bernardo Houssay 200, Ushuaia, \\ Tierra del Fuego, Argentina, tel.: 54-2901-42-2310 x162, canderson@alumni.unc.edu, ORCID: 0000-0001-8120-5689. \\ ${ }^{\mathrm{b}}$ Universidad Nacional de Tierra del Fuego (UNTDF), Instituto de Ciencias Polares, \\ Ambiente y Recursos Naturales (ICPA), Ushuaia, Tierra del Fuego, Argentina. \\ c Universidad Nacional de Tierra del Fuego (UNTDF), Instituto de Cultura, Sociedad y Estado (ICSE), \\ Ushuaia, Tierra del Fuego, Argentina. \\ ${ }^{\mathrm{d}}$ Universidad de Concepción, Facultad de Ciencias Forestales, Departamento de Manejo de Bosques y Ambiente, \\ Concepción, Chile.
}

\begin{abstract}
SUMMARY
In 2008, Argentina and Chile signed an agreement to eradicate North American beavers (Castor canadensis) and restore degraded forests. The plans and actions related to this treaty have been conducted principally by experts with biological knowledge, paying scant attention to social aspects. From a socio-ecological approach, we evaluated the perspectives of two groups of key stakeholders (managers and researchers) from Argentinean and Chilean institutions. Via surveys, we compared i) attitudes towards the binational agreement's two objectives (eradication and restoration), ii) the reasons that underlie these positions, and iii) the opinions about obstacles in implementing the agreement. The majority of both groups agreed with the two objectives, nonetheless managers supported more the eradication of the beaver and researchers supported more the restoration of degraded forests. These positions were based on biological arguments (e.g., ecological harm, being an exotic species), more than on social ones (e.g., ethics, economic impact). Paradoxically, managers and researchers identified the lack of information on social topics (e.g., internal management, interinstitutional cooperation, financing) as the main obstacle for the implementation of the agreement. While there was a high level of consensus, dissident positions were found among these stakeholders, whose profile is rather homogeneous. Managers particularly emphasized politico-institutional topics. Consequently, we are called upon to deepen and increase attention towards social dimensions in the approach to biological invasions to diversify the perspectives towards the problem, as well as to optimize and improve outcomes of potential actions in complex and heterogeneous societies.
\end{abstract}

Key words: stakeholders, exotic species, biological invasion, Patagonia.

\section{RESUMEN}

En 2008, Argentina y Chile firmaron un acuerdo para erradicar el castor norteamericano (Castor canadensis) y restaurar los bosques degradados. Los planes y acciones relacionados con dicho convenio han sido realizados principalmente por expertos con conocimientos biológicos, brindando poca atención a aspectos sociales. Desde una aproximación socio-ecológica, se evaluaron las perspectivas de dos grupos de actores sociales clave (gestores e investigadores) de instituciones argentinas y chilenas. Mediante encuestas, se compararon i) actitudes ante los dos objetivos del acuerdo binacional (erradicación y restauración), ii) fundamentos que sostienen estas posturas, y iii) opiniones sobre los obstáculos para la implementación del acuerdo. Se encontró que la mayoría de ambos grupos estuvo de acuerdo con los dos objetivos, pero los gestores apoyaron más la erradicación del castor y los investigadores más la restauración de los bosques. Estas posiciones estuvieron fundamentadas en argumentos biológicos (e.g., daño ecológico, ser una especie exótica), más que sociales (e.g., ética, impacto económico). Paradójicamente, tanto gestores como investigadores identificaron la carencia de información sobre temas sociales (e.g., gestión interna, cooperación inter-institucional, financiamiento) como un obstáculo principal para implementar el acuerdo. Si bien hubo un alto nivel de acuerdo, se encontraron posturas disidentes entre estos actores, cuyo perfil fue bastante homogéneo, y los gestores en particular enfatizaron más los temas político-institucionales. Lo anterior convoca a profundizar y aumentar la atención sobre la dimensión social en el abordaje de invasiones biológicas para diversificar tanto las perspectivas del problema, como optimizar y mejorar los resultados de posibles acciones en sociedades complejas y heterogéneas.

Palabras clave: actor, especie exótica, invasión biológica, Patagonia. 


\section{INTRODUCCIÓN}

Las invasiones biológicas constituyen una amenaza para la biodiversidad no solo al nivel global, sino también a nivel local en lugares supuestamente "prístinos", como la Patagonia austral (Anderson et al. 2006). Dentro del Cono Sur de América, la ecorregión con más especies invasoras es el remoto Bosque Subantártico. De hecho, las especies invasoras corresponden a uno de los principales factores de degradación del bosque en el Archipiélago de Tierra del Fuego, particularmente a través de los efectos de la invasión biológica del castor norteamericano (Castor canadensis Kuhl), un roedor e ingeniero de ecosistema introducido a la isla en 1946 (Anderson et al. 2009). Desde la ecología, se han documentado los impactos del castor, catalogándolo como la perturbación más extensa en los bosques subantárticos de Tierra del Fuego durante el Holoceno (Henn et al. 2016). Consecuentemente, y en parte gracias a la información disponible sobre su impacto ecológico, se firmó en 2008 el "Acuerdo entre la República Argentina y la República de Chile sobre la Restauración de los Ecosistemas Australes Afectados por el Castor Americano" (en adelante "acuerdo binacional", Malmierca et al. 2011). Sin embargo, la información científica generada hasta la fecha se centra en temas netamente biológicos mientras que tanto la invasión biológica del castor como la implementación de dicho convenio requieren de un mayor entendimiento de aspectos sociales, incluyendo temas financieros, institucionales, políticos y culturales (Anderson y Valenzuela 2014).

Es cada vez más reconocido por parte de las autoridades y la literatura científica que una gestión sustentable y una política ambiental adecuada, particularmente ligada al manejo forestal (Krott 2005, Primmer y Karppinen 2010), conllevan a la necesidad de incluir diversas perspectivas y saberes de múltiples actores sociales. Por ejemplo, la decisión de controlar una invasión biológica o restaurar un ecosistema invadido muchas veces implica acciones que generan conflictos, $y$, por lo tanto, llevar a cabo estas iniciativas requiere del involucramiento y participación de diferentes actores sociales, ya sean individuos, grupos o instituciones. De esta forma, se pueden anticipar y prevenir conflictos que de otra manera podrían terminar frenando el desarrollo de las acciones de manejo y metas propuestas (Estévez et al. 2015, Suding et al. 2015). En este sentido, para un abordaje integral de problemáticas ambientales (que son en su mayoría fenómenos socio-ambientales) es crucial involucrar en forma más estrecha a ciertos actores "clave", denominados así porque sus opiniones, actitudes e intervenciones influyen fuertemente en las decisiones políticas y/o acciones sociales con las que una comunidad responde frente a una situación ambiental determinada (Gudynas 2001). Además de los políticos y las autoridades gubernamentales, dichos actores clave pueden incluir a profesionales de la gestión y/o de la investigación de los recursos naturales que integran comités o grupos de exper- tos que diseñan e implementan los programas y políticas (Primmer y Karppiner 2010).

Coherentemente con lo anterior, la literatura científica muestra que existen discrepancias entre los conocimientos y percepciones sobre las especies invasoras entre profesionales de la conservación y otros actores sociales clave. Principalmente, estas diferencias se relacionan con las actitudes frente a opciones de manejo y técnicas de control o erradicación (e.g., rechazo ante los medios letales) (Larson 2005, García-Llorente et al. 2008). Algo similar ocurre con la restauración ecológica, la que puede ser evaluada por distintos actores de acuerdo al desenlace o resultado "final" de la intervención, como por ejemplo una actitud positiva frente al propósito de la recuperación de bosques en una zona urbana y una actitud negativa frente a los métodos de intervención para lograr esto mismo (e.g., ante el corte de árboles vivos o el uso de pesticidas) (Gobster 2000). Así, una actitud frente una especie exótica invasora no solo es determinada por el impacto de la especie en sí, sino también mediante una evaluación del efecto de la intervención para controlar dicha especie (García-Llorente et al. 2011, Gobster 2011).

En este contexto, el presente estudio aborda conceptualmente a las invasiones biológicas como sistemas socio-ecológicos, para profundizar el conocimiento de las perspectivas de dos grupos de actores clave (gestores e investigadores) en la implementación del acuerdo binacional, considerando las opciones de manejo que plantea: la erradicación del castor y la restauración de los ecosistemas degradados. Considerando el involucramiento de gestores e investigadores en la problemática, se espera encontrar un alto nivel de acuerdo entre ellos, a favor de los dos objetivos del convenio. No obstante, dadas las diferencias en los campos laborales entre ambos grupos, se espera que los gestores tengan mayor reconocimiento de los aspectos socio-políticos del acuerdo, mientras que los investigadores prioricen las dimensiones biológicas y/o limitaciones técnicas. De esta manera, mediante encuestas se comparan i) sus actitudes ante los dos componentes del acuerdo binacional (erradicación y restauración); ii) los fundamentos que sostienen sus posturas frente a estos componentes; y iii) sus opiniones sobre obstáculos a la gestión e investigación que se requiere para la implementación exitosa del acuerdo.

\section{MÉTODOS}

Caso de estudio. En 1946, veinte castores fueron introducidos en el sector argentino de Tierra del Fuego, en un intento fallido de generar una industria peletera y "enriquecer" el archipiélago con especies animales de valor comercial. En la actualidad, la población de estos mamíferos se ha expandido a más de 100.000 ejemplares en alrededor de siete millones de hectáreas. De esta superficie, tan solo en la parte argentina de la Isla Grande de Tierra del Fuego unas 30.000 hectáreas corresponden a bosque afectado por 
esta especie (Henn et al. 2016). Asimismo, la detección de su expansión al sector continental en Chile en la década del 1990 puso en manifiesto la amenaza latente para las cuencas hídricas y las actividades humanas a lo largo del bosque templado de ambos países, el cual además es el más extenso del hemisferio sur (Armesto et al. 1996).

En el año 2008, Chile y Argentina firmaron un acuerdo binacional para erradicar al castor y restaurar los ecosistemas degradados (Malmierca et al. 2011). La implementación del convenio avanzó en varios aspectos, incluyendo: 1) el financiamiento de un estudio de factibilidad para la erradicación del castor; 2) la conformación de un equipo binacional para la confección de un plan estratégico para la erradicación; y 3) la postulación y obtención individual por parte de ambos países de proyectos Global Environment Facility para ejecutar programas piloto para la erradicación.

Encuesta. Dado que las actitudes e intenciones son precursores que indican el comportamiento (Fishbein y Azjen 1975), se evaluaron estas perspectivas con respecto a la erradicación del castor y la restauración del bosque por parte de representantes de instituciones involucradas en la gestión e implementación del acuerdo binacional. Se hizo el relevamiento de datos mediante una encuesta, método ampliamente utilizado para medir percepciones de problemáticas ambientales, incluyendo las especies invasoras (e.g., García Llorente et al. 2008, 2011). En junio 2015, se invitó a participar del estudio a cuarenta y seis miembros de diez instituciones argentinas y ocho chilenas, pertenecientes a los ámbitos gubernamental, académico y privado, y de distintas escalas (local, provincial, nacional e internacional). Se tomó como criterio de selección solo las instituciones con injerencia directa sobre la toma de decisiones o que realizan investigaciones relacionadas con el manejo del castor y/o las acciones de restauración ecológica. A cada uno de los participantes se distribuyó la encuesta online en GoogleDocs vía correo electrónico con una explicación de que los datos serían utilizados como insumos en el taller de trabajo "Vinculando la investigación y la gestión del castor y la restauración ecosistémica" y en futuros proyectos de investigación relacionados a la dimensión institucional de los problemas socio-ecológicos y de restauración ecológica. A su vez, se aclaró que las respuestas eran anónimas y confidenciales. Se les reenvió la invitación a participar a quienes no respondieron en primera instancia.

La encuesta consistió en diecisiete preguntas cerradas, tres de ellas para caracterizar el perfil de los encuestados (edad, educación, institución) y catorce de elección múltiple sobre dos aspectos (las actitudes y las opiniones) que influirían en la toma de decisiones y posteriores acciones de estos informantes institucionales. Primero, se les pidió que indicaran su actitud frente las decisiones de manejo sobre la erradicación del castor y la restauración del bosque degradado. A los fines de este trabajo, se entiende por actitud la predisposición favorable o desfavorable frente a algo (Fishbein y Ajzen 1975). Por ejemplo, para la erra- dicación, se evaluó cuál era la postura (actitud) respecto a la afirmación "Los castores se deben erradicar en la Patagonia Austral” y para la restauración se usó la aseveración "Los ambientes degradados por los castores se deben restaurar”. Luego, se relevaron las opiniones sobre los factores que condicionan ciertas prácticas en la gestión. En este contexto, opinión se usa para denotar una creencia o una afirmación que incluye una evaluación subjetiva de los atributos de un objeto o un fenómeno (Fishbein y Ajzen 1975). Se consideró, además, que estas opiniones son parte de lo que se puede denominar "opinión experta", pues son las apreciaciones y experiencias del trabajo de los actores sociales involucrados directamente en el manejo de recursos naturales, la fauna y el bosque. Específicamente, se evaluó la opinión de los participantes sobre los obstáculos para la implementación de programas de erradicación de especies invasoras y restauración ecosistémica, y sobre el conocimiento que consideran escaso o carente para la implementación del acuerdo binacional.

Análisis. Se caracterizó la población encuestada, evaluando la tasa de respuesta $\left(\mathrm{n}^{\circ}\right.$ de encuestas completadas $/ \mathrm{n}^{\circ}$ de encuestas enviadas) y una descripción por grupo: i) encuestados asociados a la gestión versus ii) encuestados asociados a la investigación. Luego, se caracterizaron por grupo las actitudes frente las decisiones de manejo como porcentaje "en favor", "en contra" y "no sé". Los fundamentos para estas actitudes fueron analizados a través del cálculo de la prominencia ( $S$, por "salience" en inglés) de cada razón por todos versus cada grupo, con una adaptación del índice de Sutrop (2001) [fórmula 1]:

$$
S=f(r)
$$

Donde:

$(f)=$ frecuencia: es la cantidad de veces que una razón fue seleccionada por los encuestados dividida por el número total de encuestas.

$(r)=$ ranking: es la posición de esa razón relativa al número de menciones de las demás.

Las respuestas ante las preguntas de las opciones sobre posibles obstáculos fueron clasificadas por su relación con i) gestión interna de la institución, ii) cooperación interinstitucional, iii) financiamiento, iv) aspectos políticonormativos y v) temas aplicados (incluyendo técnicas de caza). Luego, los tipos de conocimiento que requieren mayor investigación para afrontar la problemática fueron organizados como i) institucionales, ii) socio-culturales, iii) económicos, iv) político-normativos, v) biológicos, vi) consideraciones aplicadas y vii) ya existe información suficiente. Finalmente, se calculó un valor de importancia (VI) [fórmula 2] por cada categoría para describir la importancia de una opinión en comparación a las demás, donde:

$$
V I=f R+a R
$$


La frecuencia relativa ( $f R$ ) de una categoría fue calculada como el número de encuestas con esa categoría dividido por el número total de encuestas. La abundancia relativa $(a R)$ de una categoría era el número de veces que fue seleccionada una respuesta dividido por el número total de posibles respuestas. Dado que con estas preguntas el encuestado podría elegir entre 0-3 o 0-4 respuestas, surgió un total de 30 o 40 posibilidades. El valor máximo del VI es 2.

\section{RESULTADOS}

Perfil de los encuestados. Del total de cuarenta y seis invitaciones a representantes institucionales, veinticuatro completaron la encuesta $(52,2 \%)$. Hubo igual participación entre representantes de instituciones de investigación y de gestión (doce cada uno), y el muestreo incluyó instituciones a nivel local, provincial, nacional e internacional. De estas instituciones, el 54,2 \% pertenecen a organismos nacionales argentinos, pero con presencia y relativa autonomía en el territorio fueguino (Centro Austral de Investigaciones Científicas [perteneciente del Consejo Nacional de Investigaciones Científicas y Técnicas] y las oficinas locales de la Administración de Parques Nacionales y del Instituto Nacional de Tecnología Agropecuaria). El porcentaje restante se divide en i) 16,7 \% instituciones provinciales argentinas (Secretaría de Ambiente, Desarrollo Sostenible y Cambio Global y consultores independientes), ii) $12,5 \%$ instituciones argentinas sin sede en Tierra del Fuego (Sede Central-Administración de Parques Nacionales, Ministerio de Agricultura, Universidad Nacional de la Plata), iii) 12,5 \% instituciones chilenas (Servicio Agrícola y Ganadero, Pontificia Universidad Católica de Chile, consultores privados), y iv) 4,2 \% instituciones internacionales (United Nations Food and Agriculture Organization).

Actitudes y fundamentos sobre las opciones de manejo expresadas en el acuerdo binacional. Se encontró que la gran mayoría ( $80 \%$ ) de los encuestados estaban de acuerdo con las decisiones de erradicar el castor y de restaurar los ecosistemas degradados (cuadro 1). No obstante, se detectó una diferencia de énfasis entre los dos grupos, siendo los gestores más enfáticos en el apoyo a la erradicación del castor y los investigadores a la restauración de los ecosistemas degradados.

También, hubo un consenso general que las principales razones que fundamentan la erradicación de los castores son ecológicas. En el caso de ambos grupos, la afirmación más prominente se vincula con el impacto ecológico que provoca el castor en Tierra del Fuego, seguido por otros fundamentos ecológicos relacionados con los ecosistemas forestales fueguinos, mientras que las razones éticas o socio-económicas fueron mucho menores (cuadro 2a). En contraste, para justificar el apoyo a la restauración ecosistémica, los encuestados se basaron en fundamentos sociales y económicos. Las razones biológicas -al contrario de la erradicación- fueron menores, pero los fundamentos
Cuadro 1. Actitudes de dos grupos de actores clave (Gestores e Investigadores) ante el Acuerdo Binacional (Argentina-Chile) sobre la erradicación del castor y la restauración de ecosistemas degradados.

Attitudes of two groups of key stakeholders (managers and researchers) on the binational agreement (Argentina-Chile) to eradicate beavers and restore degraded ecosystems.

\begin{tabular}{llrrc}
\hline \multirow{2}{*}{ En favor de: } & & \multicolumn{3}{c}{ Porcentaje de actores (\%) } \\
\cline { 3 - 5 } & & Todos & Gestores & Investigadores \\
\hline \multirow{3}{*}{ Erradicación } & 79,2 & 91,7 & 66,7 \\
& No & 16,7 & 8,3 & 25,0 \\
& No sé & 4,2 & 0 & 8,3 \\
\hline \multirow{2}{*}{ Restauración } & Sí & 83,3 & 75,0 & 91,7 \\
& No & 8,3 & 16,6 & 0 \\
& No sé & 8,3 & 8,3 & 8,3 \\
\hline
\end{tabular}

éticos siguieron siendo de una importancia mínima para los expertos en general (cuadro 2b).

Cabe destacar que solo cuatro encuestados estuvieron en contra de la erradicación (uno gestor y tres investigadores). Basaron su argumento en i) la imposibilidad de la erradicación y ii) la naturalización del castor en Tierra del Fuego. Por otro lado, los actores en desacuerdo con la restauración (dos gestores) fundamentaron sus decisiones en términos de i) costo-beneficio en el gasto de estas iniciativas, considerando ii) la "auto-recuperación” de los ecosistemas afectados.

Opiniones sobre obstáculos y necesidades para la implementación del acuerdo binacional. Ambos grupos encuestados (gestores e investigadores) tuvieron opiniones muy similares e identificaron que el principal obstáculo para la implementación de un plan tendiente a erradicar el castor es la gestión interna de las instituciones, seguido por la escasa cooperación inter-institucional (cuadro 3a). En relación a la restauración, los informantes reconocen nuevamente la baja cooperación inter-institucional, pero ante esta pregunta la categoría de gestión interna bajó al último lugar de importancia. Una diferencia notable entre los dos grupos fue la categoría de aspectos político-normativos que estuvo en $2^{\circ}$ lugar para los gestores, pero fue el obstáculo menos importante para los investigadores (cuadro $3 \mathrm{~b}$ ), demostrando así diferencias de competencias profesionales que afectan las perspectivas de los dos grupos de expertos.

Por otra parte, ambos grupos coincidieron en la necesidad de generar más información para implementar las acciones propuestas en el acuerdo binacional (en ambos casos la categoría "ya existe información suficiente" fue la menos seleccionada). No obstante, hubo diferencias entre los ges- 
Cuadro 2. Fundamentos de las actitudes de los actores clave (Gestores e Investigadores) ante el Acuerdo Binacional (Argentina-Chile) sobre la (a) erradicación del castor y la (b) restauración de ecosistemas degradados.

Reasons underling the attitudes of key stakeholders (managers and researchers) regarding the binational agreement (Argentina-Chile) to (a) eradicate beavers and (b) restore degraded ecosystems.

\begin{tabular}{|c|c|c|c|}
\hline \multirow[b]{2}{*}{ (a) Fundamentos para la erradicación del castor } & \multicolumn{3}{|c|}{ Prominencia (Rank) } \\
\hline & Todos & Gestores & Investigadores \\
\hline $\begin{array}{l}\text { Están actualmente causando daños significativos a los sistemas ecológi- } \\
\text { cos de Tierra del Fuego }\end{array}$ & $0,63(1)$ & $0,83(1)$ & $0,50(1)$ \\
\hline $\begin{array}{l}\text { Amenazan a futuro los sistemas ecológicos más al norte mientras siguen } \\
\text { expandiendo }\end{array}$ & $0,27(2)$ & $0,33(2)$ & $0,21(2)$ \\
\hline Son especies no-nativas & $0,14(3)$ & $0,19(3)$ & $0,08(3)$ \\
\hline Es nuestra responsabilidad ética & $0,05(4)$ & $0,08(4)$ & $0,01(6)$ \\
\hline $\begin{array}{l}\text { Están actualmente causando daños significativos a los sistemas econó- } \\
\text { micos de Tierra del Fuego }\end{array}$ & $0,03(5)$ & $0,02(5)$ & $0,08(4)$ \\
\hline $\begin{array}{l}\text { Amenazan a futuro los sistemas económicos más al norte mientras si- } \\
\text { guen expandiendo }\end{array}$ & $0,01(6)$ & $0,00(6)$ & $0,03(5)$ \\
\hline \multicolumn{4}{|l|}{ (b) Fundamentos para la restauración ecológica } \\
\hline La restauración ecológica también trae beneficios económicos y sociales & $0,67(1)$ & $0,67(1)$ & $0,67(1)$ \\
\hline Los bosques fueguinos proveen servicios ecosistémicos importantes & $0,29(2)$ & $0,67(2)$ & $0,25(2)$ \\
\hline Para preservar la flora y fauna autóctona & $0,10(3)$ & $0,02(4)$ & $0,25(2)$ \\
\hline Es nuestra responsabilidad ética & $0,05(4)$ & $0,08(3)$ & $0,03(5)$ \\
\hline Los bosques fueguinos son los más australes y más prístinos del mundo & $0,03(5)$ & $0,00(5)$ & $0,06(4)$ \\
\hline
\end{tabular}

Se presentan datos sobre la prominencia, $S$, adaptando el índice de Sutrop (2001), para cada fundamento y su (lugar) en el ranking en orden descendente de acuerdo a prominencia.

Salience data are presented, S, adapting Sutrop's (2001) index, for each reason and its (place) in the ranking in descending order of salience.

Cuadro 3. Principales obstáculos identificados por actores clave (Gestores e Investigadores) ante el Acuerdo Binacional (ArgentinaChile) sobre la (a) erradicación del castor y (b) la restauración de ecosistemas degradados.

Main obstacles identified by key stakeholders (managers and researchers) regarding the binational agreement (Argentina-Chile) to (a) eradicate and (b) restore degraded ecosystems.

\begin{tabular}{|c|c|c|c|}
\hline \multirow{2}{*}{$\begin{array}{l}\text { Principales obstáculos } \\
\text { (a) Para la erradicación del castor }\end{array}$} & \multicolumn{3}{|c|}{ Valor de importancia (Rank) } \\
\hline & Todos & Gestores & Investigadores \\
\hline Gestión interna & $0,84(1)$ & $0,85(1)$ & $0,84(1)$ \\
\hline Cooperación inter-institucional & $0,79(2)$ & $0,76(2)$ & $0,81(2)$ \\
\hline Financiamiento & $0,73(3)$ & $0,68(3)$ & $0,78(3)$ \\
\hline Contexto político-normativo & $0,59(4)$ & $0,68(4)$ & $0,50(5)$ \\
\hline Capacidad técnica & $0,51(5)$ & $0,45(5)$ & $0,56(4)$ \\
\hline \multicolumn{4}{|l|}{ (b) Para la restauración ecosistémica } \\
\hline Cooperación inter-institucional & $1,08(1)$ & $1,07(1)$ & $1,08(1)$ \\
\hline Financiamiento & $0,79(2)$ & $0,80(2)$ & $0,78(2)$ \\
\hline Contexto político-normativo & $0,62(3)$ & $0,80(2)$ & $0,44(5)$ \\
\hline Capacidad técnica & $0,45(4)$ & $0,23(4)$ & $0,67(3)$ \\
\hline Gestión interna & $0,41(5)$ & $0,23(4)$ & $0,58(4)$ \\
\hline
\end{tabular}

Se presentan los resultados de la importancia por categoría y su (lugar) en el ranking en orden descendente de acuerdo a los valores de importancia. Results are presented by the importance per category and the (place) in the ranking in descending order of importance values. 
tores y los investigadores sobre los temas de conocimiento que requieren mayor investigación (cuadro 4). En general, para la erradicación del castor, los vacíos en información percibidos fueron aquellos relacionados con aspectos técnicos (e.g., el trampeo) y le siguieron información relacionada con la economía y aspectos político-normativos para el financiamiento, y la gestión de las acciones del acuerdo. Los temas dentro de la biología fueron considerados de menor importancia por ambos grupos para la erradicación, pero fueron más prioritarios para la restauración (cuadro 4b). A su vez, los expertos señalaron que otros déficits de información para la restauración son aquellos relacionados a los aspectos político-institucionales y económicos.

\section{DISCUSIÓN}

Implicancias para el manejo de invasiones biológicas. Las acciones de manejo ambiental, particularmente para las especies exóticas invasoras, están mediadas por factores ambientales, aspectos sociales y la interacción entre ambos (Gobster 2011). En este sentido, destacamos que a partir de la firma del convenio binacional entre Argentina y Chile para la erradicación del castor y la restauración de los eco- sistemas degradados (Malmierca et al. 2011), han aumentado los estudios que buscan aportar este tipo de información multi-dimensional sobre el proceso de invasión de esta especie exótica, no solo desde lo ecológico (e.g., ocupación según tiempo de colonización, Davis et al. 2016; invasión de la estepa, Pietrek y González-Roglich 2015) sino también desde lo práctico (e.g., la restauración activa con ensayos de plantación de lenga [Nothofagus pumilio (Poepp. et Endl.) Krasser], Henn et al. [2014]) y lo social. Con respecto a este último tema, se ha evaluado también la disposición a pagar para la restauración por parte de los residentes de la región de Magallanes (Chile) (Soto Simeone y Soza-Amigo 2014) y se ha caracterizada la perspectiva de estancieros sobre la estructura de posibles programas e incentivos para la erradicación y restauración (Santo et al. 2015).

Específicamente, estos resultados constituyen la primera descripción de las posturas de los representantes de las instituciones con gerencia en la implementación del convenio binacional, lo cual permite empezar a relacionar dichos aspectos con el proceso socio-político que excede el ámbito netamente científico. En este sentido, incluir la perspectiva de los "expertos" es relevante porque sus acciones se relacionan directamente con la implementación

Cuadro 4. Temas prioritarios de investigación para la implementación del Acuerdo Binacional (Argentina-Chile) para la (a) erradicación del castor y (b) la restauración de ecosistemas degradados, según los actores clave (Gestores e Investigadores).

Priority research topics for the implementation of the binational agreement (Argentina-Chile) to (a) eradicate beavers and (b) restore degraded ecosystems, according to key stakeholders (managers and researchers).

\begin{tabular}{lcccc}
\hline \multicolumn{1}{c}{ Prioridades de investigación } & & \multicolumn{2}{c}{ Valor de importancia (Rank) } \\
\cline { 1 - 2 } \cline { 4 - 4 } Aspectos aplicados/técnicos & & Todos & Gestores & Investigadores \\
Economía & & $0,84(1)$ & $0,70(3)$ & $0,99(1)$ \\
Político-normativa & $0,70(2)$ & $0,80(1)$ & $0,60(2)$ \\
Socio-cultural & $0,70(2)$ & $0,80(1)$ & $0,60(2)$ \\
Institucional & $0,43(4)$ & $0,46(4)$ & $0,39(5)$ \\
Biología & $0,35(5)$ & $0,23(5)$ & $0,48(4)$ \\
Ya hay información suficiente & $0,12(6)$ & $0,23(5)$ & $0,00(7)$ \\
\hline$\quad$ (b) Para la restauración ecosistémica & $0,12(6)$ & $0,11(7)$ & $0,12(6)$ \\
\hline Biología & & & $1,13(1)$ \\
Político-normativa & $0,98(1)$ & $0,84(2)$ & $0,47(4)$ \\
Institucional & $0,70(2)$ & $0,92(1)$ & $0,59(2)$ \\
Economía & $0,58(3)$ & $0,58(3)$ & $0,59(2)$ \\
Socio-cultural & $0,53(4)$ & $0,46(4)$ & $0,24(5)$ \\
Ya hay información suficiente & $0,35(5)$ & $0,46(4)$ & $0,12(6)$ \\
\hline
\end{tabular}

Se presentan los resultados de la importancia por categoría y su (lugar) en el ranking en orden descendente. Results are presented for the importance per category and its (place) in the ranking in descending order. 
y el eventual éxito del documento político (Primmer y Karppinen 2010). Cabe señalar que en otros estudios (e.g., García Llorente et al. 2008 en España) se ha mostrado que la actitud de actores involucrados en las invasiones biológicas, tanto hacia la especie como hacia su manejo, está condicionado en parte por los impactos de la especie. Por ende, en el caso del castor se esperaría que dado sus impactos visibles y dramáticos (Henn et al. 2016), otros grupos sociales, además de los gestores y científicos, llegaran a percibir este problema y tener actitudes y opiniones que apoyan el convenio binacional y sus objetivos. No obstante, es sabido que en general los especialistas (e.g., profesionales de la conservación) están más dispuesto a apoyar estas iniciativas, pero el nivel de apoyo o de aceptación por parte de otros actores también puede ser alto, dependiendo de las características personales, como su interés en la naturaleza y el conocimiento sobre las especies exóticas invasoras (García Llorente et al. 2011).

Los bosques subantárticos como sistemas socio-ecológicos. La Patagonia austral cuenta con un alto porcentaje de áreas naturales protegidas y la ecorregión de los bosques subantárticos es catalogada como una de las más prístinas del planeta (Mittermeier et al. 2003). Sin embargo, recientemente ha sido reconocido como la que también posee la mayor invasión de mamíferos exóticos, en términos relativos y en números absolutos, en todo Argentina, Chile y Uruguay (Ballari et al. 2016). Dentro del ensamble de estas especies invasoras, el castor americano en Tierra del Fuego es emblemático por su extenso impacto ecológico en el bosque y por estar incorporado en los procesos socio-políticos y concepciones simbólicas de la sociedad. De esta forma, el caso además provee una lección general, ya que pone en evidencia que la gestión y conservación de estos bosques necesita de una mayor incorporación de las dimensiones sociales y políticas. Así, aporta no solo al entendimiento del fenómeno de las invasiones biológicas sino remarca que incluso los ecosistemas ubicados en sitios remotos y supuestamente "silvestres" requieren de un abordaje socio-ecológico para su manejo.

Con respecto a la gestión de estos socio-ecosistemas, en base a estos resultados, se puede decir que si bien hay un alto nivel de consenso entre los gestores e investigadores involucrados en la temática para accionar de acuerdo a la propuesta del convenio binacional, no es un acuerdo absoluto e incluso en este grupo relativamente homogéneo existen discrepancias. Dado lo anterior, se debería tomar en cuenta que las expectativas sociales del manejo ambiental de estos bosques son diversas. Pero más que enfatizar las diferencias de opinión, este dato es útil para que internamente se reconozcan las distintas posturas entre los participantes, lo cual permite crear procesos de construcción colectiva del conocimiento y de gestión que tomen en cuenta y respeten las coincidencias y también las diferencias de valores, creencias y actitudes. No considerar esta pluralidad de valores podría generar conflictos, especial- mente entre especialistas (científicos y gestores) y otros actores sociales (Estévez et al. 2015).

\section{CONCLUSIONES}

En consonancia con la hipótesis original, se encontró un alto nivel de consenso por parte de ambos grupos con respecto a su acuerdo frente los dos objetivos del convenio binacional (erradicación del castor y restauración de los ecosistemas afectados), pero también efectivamente existieron leves diferencias entre los dos grupos en la fundamentación de sus opiniones con respecto a la problemática en sí y las limitaciones para lograr la implementación del acuerdo. Pensando hacia el futuro, esta información ayudaría a orientar nuevos esfuerzos tanto por parte de los científicos como por las agencias de financiamiento para paliar los obstáculos presentados al momento de ejecutar acciones tendientes a la erradicación y la restauración. Se destaca la necesidad de abordar temáticas del ámbito institucional, incluyendo la colaboración entre actores, la organización interna, los vínculos interinstitucionales. También, sería importante realizar nuevas investigaciones sobre otros actores que pueden ser clave en otros aspectos del éxito de programas de manejo ambiental. Por ejemplo, este estudio se enfoca en los gestores e investigadores, debido a su involucramiento en la implementación práctica del acuerdo binacional, pero otros actores (e.g., organizaciones no gubernamentales ambientales, asociaciones, fundaciones, otras instituciones nacionales vinculadas indirectamente) son potencialmente relevantes para generar el apoyo político y social que determinan el contexto propicio necesario para intervenciones de manejo, como la erradicación y la restauración. Finalmente, este estudio constituye una línea base antes de la implementación de los dos proyectos Global Environment Facility en la región (uno en Argentina y otro en Chile) que comenzaron formalmente en la primavera de 2015. Este hecho podría constituirse en un hito histórico en el proceso de mitigación de una problemática ambiental y posterior restauración del ambiente degradado. Por lo tanto, se propone que las próximas investigaciones tengan en consideración las acciones y relaciones que se van generando entre las instituciones y actores encargados de la ejecución de mencionados proyectos.

\section{AGRADECIMIENTOS}

El presente estudio se hizo en el contexto del taller "Vinculando la Investigación y la Gestión del Castor y la Restauración Ecosistémica", financiado por el proyecto ECO-Link (NSF grant \#GEO 1262148) y realizado en coordinación con G. Martínez Pastur, R. Soler (CADICCONICET) y L. Malmierca (Administración de Parques Nacionales). CR y JCP fueron apoyados con una beca doctoral cofinanciada CONICET-UNTDF y una beca postdoctoral latinoamericana CONICET, respectivamente. 


\section{REFERENCIAS}

Anderson CB, AEJ Valenzuela. 2014. Do what I say, not what I do. Are we linking research and decision-making about invasive species in Patagonia? Ecología Austral 24(2): 193-202.

Anderson CB, R Rozzi, JC Torres-Mura, SM McGehee, MF Sherriffs, E Schuettler, AD Rosemond. 2006. Exotic vertebrate fauna in the remote and pristine sub-Antarctic Cape Horn Archipelago region of Chile. Biodiversity and Conservation 15(10): 3295-3313.

Anderson CB, G Martínez Pastur, MV Lencinas, PK Wallem, MC Moorman, AD Rosemond. 2009. Do introduced North American beavers engineer differently in southern South America?-An overview with implications for restoration. Mammal Review 39(1): 33-52.

Armesto JJ, C Villagrán, MK Arroyo. 1996. Ecología de los bosques nativos de Chile. Santiago, Chile. Editorial Universitaria. $470 \mathrm{p}$.

Ballari SA, CB Anderson, AEJ Valenzuela. 2016. Invasive exotic mammal research and management in the Southern Cone. Mammal Review 46(3): 229-240.

Boon TE, H Meilby. 2007. Describing management attitudes to guide forest policy implementation. Small-scale Forestry 6(1): 79-92.

Davis E, AEJ Valenzuela, S Murcia, CB Anderson. 2016. Habitat selection by introduced North American beavers, Castor canadensis, in southern Patagonia. Mastozoologia Neotropical 23(1): 51-61.

Estévez R, CB Anderson, JC Pizarro, M Burgman. 2015. Clarifying values, risk perception and attitudes to resolve or avoid social conflicts in invasive species management. Conservation Biology 29(1): 19-30.

Fishbein M, I Ajzen. 1975. Belief, attitude, intention, and behavior: an introduction to theory and research. Reading, MA, USA. Addison-Wesley. 575 p.

García-Llorente M, B Martín-López, JA Gónzalez, P Alcorlo, C Montés. 2008. Social perceptions of the impacts and benefits of invasive alien species: implications for management. Biological Conservation 141(12): 2969-2983.

García-Llorente M, B Martín-López, P Nunes, JA González, P Alcorlo, C Montes. 2011. Analyzing the social factors that influence willingness to pay for invasive alien species management under two different strategies: eradication and prevention. Environmental Management 48(3): 418-435.

Gobster PH. 2000. Restoring nature: Human actions, interactions, and reactions. In Gobster PH y RB Hull eds. Restoring nature: perspectives from the social sciences. Washington DC. USA. Island Press. p. 1-19.
Gobster PH. 2011. Factors affecting people's responses to invasive species: animals, human perceptions, attitudes and approaches to management. In Rotherhman ID, RA Lambert eds. Invasive and introduced plants and management. Washington DC, USA. Earthscan Publishers. p. 249-263.

Gudynas E. 2001. Actores sociales y ámbitos de construcción de políticas ambientales. Ambiente \& Sociedade 4(8): 5-19.

Henn JJ, CB Anderson, G Kreps, MV Lencinas, R Soler, G Martínez Pastur. 2014. Determining abiotic and biotic factors that limit transplanted Nothofagus pumilio seedling success in abandoned beaver meadows in Tierra del Fuego. Ecological Restoration 32(4): 369-378.

Henn JJ, CB Anderson, G Martinez Pastur. 2016. Landscape-level impact and habitat factors that explain invasive beaver distribution in Tierra del Fuego. Biological Invasions 18(6): 1679-1688.

Krott M. 2005. Forest policy analysis. Page European Forest Institute. Dordrecht, The Netherlands. Springer. 323 p.

Larson BMH. 2005. The war of the roses: demilitarizing invasion biology. Frontiers in Ecology and the Environment 3(9): 495-500.

Malmierca L, MF Menvielle, D Ramadori, B Saavedra, A Saunders, N Soto-Volkart. 2011. Eradication of beaver (Castor canadensis), an ecosystem engineer and threat to southern Patagonia. In Veitch R, MN Clout MN, DR Towns eds. Island invasives: eradication and management. Gland, Switzerland. IUCN. p. 87-90.

Mittermeier R, C Mittermeier, TM Brooks, JD Pilgram, WR Konstant, GAB da Fonseca, C Kormos. 2003. Wilderness and biodiversity conservation. PNAS 100(18):1030910313.

Pietrek AG, M González-Roglich. 2015. Post-establishment changes in habitat selection by an invasive species: beavers in the Patagonian steppe. Biological Invasions 17(11): 3225-3235.

Primmer E, H Karppinen. 2010. Professional judgment in nonindustrial private forestry: Forester attitudes and social norms influencing biodiversity conservation. Forest Policy and Economics 12(2): 136-146.

Santo AR, MG Sorice, CJ Donlan, CT Franck, CB Anderson. 2015. A human-centered approach to designing invasive species eradication programs on human-inhabited islands. Global Environmental Change 35: 289-298.

Soto Simeone A, S Soza-Amigo. 2014. Valoración económica del bosque nativo afectado por la introducción del castor americano en Tierra del Fuego. Bosque 35(2): 229-234.

Sutrop U. 2001. List task and a cognitive salience index. Field Methods 13(3): 263-276. 